\title{
¿Son necesarias las sujeciones físicas en personas mayores como medida de prevención de caídas?
}

\author{
Lidia Sánchez-Prieto ${ }^{1}$, María Teresa Coll ${ }^{2}$, \\ María del Carmen Orte ${ }^{1}$, Margarita Vives ${ }^{1}$, y Joana Da Gama ${ }^{3}$ \\ ${ }^{1}$ Universidad de las Islas Baleares (España); ${ }^{2}$ Directora área de personas mayores. \\ INTRESS; ${ }^{3}$ Universidade do Porto (Portugal)
}

En los últimos años se está apostando por medidas alternativas a las sujeciones físicas como medidas preventivas de las caídas en personas mayores. El motivo principal es que las consecuencias de las sujeciones físicas son severas, tanto a nivel físico como psicológico en las personas mayores. Además, se postula que la retirada de sujeciones no implicará tantas caídas o efectos negativos como se ha ido defendiendo hasta la actualidad. Por consiguiente, se estudió la afectación que implicará la retirada de sujeciones en las personas mayores del centro residencial de Ferrerías, gestionado por INTRESS. La muestra se compuso de un total de 19 usuarios, de los que se realizó un seguimiento durante 12 meses desde la retirada de las sujeciones físicas. El estudio recoge el número de caídas de los usuarios, las consecuencias y los motivos de las caídas. Además, explica las medidas alternativas establecidas por el centro residencial y que son defendidas como las estrategias adecuadas para la prevención de caídas. La futura normativa y legislación debe apostar por la eliminación de las restricciones físicas, desarrollando medidas que respeten la calidad de vida de la persona y su bienestar emocional.

Palabras clave: Sujeciones físicas, medidas alternativas, centro residencial, calidad de vida, personas mayores.

Are physical restraints in older people necessary as a fall prevention measure? In recent years, it is betting on alternative measures to physical restraints as preventive measures of falls in the elderly. Main reason is that consequences of physical restraints are severe, both physically and psychologically in elderly people. In addition, it is postulated that remove restraints will not imply as many falls or negative effects as has been defended until today. Consequently, affectation that will involve the removal of restraints in the elderly from the Ferrerías residential center, managed by INTRESS, was studied. The sample consisted of a total of 19 users, who were followed after the removal of physical restrictions for 12 months. Study includes user number falls, consequences and reasons for the falls. In addition, it explains the alternative measures established by the residential center and that are defended as the appropriate strategies for the prevention of falls. The future regulations and legislation must focus on the elimination of physical restrictions. It would be necessary develop measures that respect the person's quality of life and emotional well-being.

Keywords: Physical restraints, alternative measures, residential center, quality of life, elderly people.

Correspondencia: Lidia Sánchez-Prieto. Universidad de las Islas Baleares. Departamento de pedagogía y didácticas específicas. Ctra. De Valldemossa s/n. C.P.: 07122. Palma, Illes Balears (España).E-mail: lydia.sanchez@uib.es 
Durante muchos años, se ha apostado por las sujeciones físicas de las personas mayores como una medida para evitar las caídas de éstas. Como señalan Villacieros, Steegman, Mejías, y Bermejo (2013), esa medida se establecía como estrategia, para prevenir caídas, las consecuencias o las lesiones que se podrían asociar, e incluso, posibles deambulaciones que desarrollaran. Las prevalencias registradas por los estudios son diversas, pero, con frecuencia son cifras elevadas. Sánchez et al. (2004) indicaban que un 17,9\% de la población de 65 o más años española había padecido una caída en los últimos 12 meses. Santamaría, Giménez, Satorra, Orrio, y Montoy (2015) señalaban que 1 de cada 4 personas de 75 o más años había padecido una caída en el último año, es decir, un 25\% de la población encuestada.

La prevalencia internacional puede estimarse que oscila entre el $7 \%$ y el $12 \%$ En el estudio de Carballo, Gómez, Casado, Ordás, y Fernández (2018), la prevalencia en personas mayores institucionalizadas fue del $32 \%$, subrayando que la prevalencia de caídas de personas mayores en los servicios sociosanitarios suele ser más elevada que en su propio entorno.

La Organización Mundial de la Salud (OMS) (2008) anunciaba que anualmente se producen 646000 caídas mortales. Por lo que, las caídas forman parte de una realidad de las personas mayores, asociándose, incluso, a consecuencias graves. Por consiguiente, se debe actuar para prevenir esas posibles secuelas o consecuencias. Como se explicaba anteriormente, las sujeciones físicas se convertían en el recurso utilizado como medida de prevención.

Sin embargo, en los últimos años, se ha generado un debate asociado a si el uso de restricción física es adecuado, e incluso, si es ético. Como subrayan Briones y Rodríguez (2017), las sujeciones físicas y químicas son valoradas como una forma de vulnerabilidad hacia los derechos de las personas mayores. Esta estrategia actuará como una potencial limitación del principio de autonomía al restringir la libertad de las personas mayores (Briones y Rodríguez, 2017; Villacieros et al., 2013). Hamers y Huizing (2005) explican que la restricción de la libertad sólo podría ser justificada a partir del principio de beneficencia, de manera que, esa medida suponga un beneficio para la persona a la que se le aplica. Precisamente, asociado al razonamiento de los autores, se plantea el debate, debido a que, las investigaciones parecen dudar de los beneficios que suponen las sujeciones para las personas mayores. Para empezar, se han registrado consecuencias relacionadas con el uso frecuente de restricciones físicas (Hamers y Huizing, 2005). Entre esas repercusiones se pueden señalar infecciones, incremento del deterioro funcional, problemáticas psicológicas, debilitación muscular o aparición de incontinencias (Tortosa, Granell, Fuenmayor, y Martínez, 2016; Villacieros et al., 2013).

Las problemáticas mentales, cognitivas o psicológicas también se han indicado como posibles consecuencias de las restricciones físicas, destacando un aumento en el deterioro de algunas capacidades cognitivas como la memoria o el lenguaje (De Bellis et 
al., 2013). Además, se señala que las sujeciones físicas se establecen como medida de intervención o de prevención ante las caídas, pero que, en realidad, no son las actuaciones adecuadas. Actuarán como mecanismos que encubren o enmascaran las necesidades reales y que deberían ser solucionados, tales como la falta de personal en las instituciones, la sobrecarga de los cuidadores, los problemas de equilibrio de personas mayores, osteoporosis, entre otros (Burgueño y Heras, 2017; Villacieros et al., 2013). Además, Mion et al. (2011) confirman que, la mayoría de profesionales, presentan actitudes contrarias al uso de restricciones, valoran que, en situaciones delicadas, pueden llegar a ser necesarias.

En cambio, empiezan a emerger planes alternativos para la prevención de caídas que no suponen un riesgo para la integridad de la persona. Las estrategias alternativas más utilizadas son el uso de camas ajustables, de dispositivos para el movimiento, uso de calzado antideslizante, protectores de caderas, entre otros. En definitiva, se apuesta y se fomentan los planes de evaluación y de gestión de caídas individualizados (Mion et al., 2011; Tortosa et al., 2016). Esos planes individualizados de prevención de caídas deben de representar las nuevas medidas asumidas, tanto por las autoridades responsables, como por los profesionales que trabajan con las personas mayores. Para su posible aplicación, también se debe de tener en cuenta que será necesario la retirada de las sujeciones en residencias y centros de día donde todavía se aplican a día de hoy (Burgueño y Heras, 2017; Villacieros et al., 2013).

Siguiendo el anterior planteamiento, en el centro residencial de Ferreries (gestionado por Intress), se planteó la eliminación de las restricciones físicas y la substitución por planes de prevención de caídas individualizados. Por lo tanto, el objetivo general de este estudio era comprobar si el uso de la retirada de sujeciones físicas provocaba múltiples caídas de los usuarios del centro residencial. En concreto, el estudio pretendía determinar: Si la retirada de sujeciones físicas implicaba la aparición de múltiples caídas de los usuarios del centro residencial. Si las caídas provocaban consecuencias graves. Las características asociadas a las caídas de los usuarios del centro residencial.

\section{MÉTODO}

Se realizó un estudio descriptivo, longitudinal en el centro residencial de Ferrerías (Islas Baleares). Intress, organismo que gestiona el centro residencial, puso en marcha un programa de eliminación de las contenciones a partir del estudio de medidas alternativas, individualizadas y personalizadas. El estudio se estableció en el periodo que oscilaba entre los años 2017 y 2018. 


\section{Participantes}

Se llevó a cabo un muestreo no probabilístico, accidental. La muestra utilizada se correspondía con la población del estudio-usuarios del centro residencial de Ferrerías. Hubo un total de 19 usuarios durante el año 2017.

Se seleccionaron sólo aquellos usuarios que cumplían los siguientes criterios de inclusión: a) eran usuarios que presentaban tendencia a las caídas, b) se les había retirado las sujeciones físicas y se había sustituido por planes de prevención de caídas individualizados y c) se les había realizado seguimiento del número de caídas durante los últimos 12 meses (2018). La edad media de los participantes fue de 87,65 años, presentando una desviación estándar de 8,31 años. En la muestra, predominaban las mujeres, identificándose un total de 70,6\%. Como puede verse en la Tabla 1, el grupo de edad más predominante eran las mujeres con 85 o más años $(47,1 \%)$. Respecto a los hombres, también se identificaba un mayor porcentaje en aquellos que tenían 85 o más años.

Tabla 1. Porcentajes de la muestra por edades y sexo

\begin{tabular}{lcccr}
\hline & Entre 65 y 74 años & Entre 75 y 84 años & 85 o más años & Total \\
\hline Hombres & 59 & 5,9 & 176 & 29,4 \\
\hline Mujeres & 59 & 176 & 471 & 70,6 \\
\hline Total & 11,8 & 23,5 & 64,7 & 100 \\
\hline
\end{tabular}

\section{Procedimiento}

El proceso de eliminación de las sujeciones se caracterizó por el desarrollo de diferentes fases, siguiendo las recomendaciones establecidas por CEOMA:

Fase inicial: formación, diagnóstico inicial, sistematización de la información, valoración e implicación de profesionales.

Fase de implantación: valoración y evaluación de cada uno de los casos, planificación y consenso de las posibles intervenciones, desarrollo de pautas de intervención, implantación de nuevos protocolos de caídas.

Fase de evaluación: evaluación, medida de los resultados y diagnóstico final.

El proceso de eliminación tuvo una duración de dos años; en el primer año se desarrolló la implementación de las medidas alternativas y en el segundo año se apostó por asegurar una buena práctica.

\section{Recogida de datos}

El estudio se establece a partir del análisis de datos obtenidos a partir de la base de datos del programa Aegerus-sistema de información especializado en residencias asistidas y sociosantiarias. Las variables analizadas fueron: número de caídas, causas de las caídas (factores extrínsecos e intrínsecos), diagnóstico, tratamiento que recibían, consecuencias tras la caída, medidas preventivas asumidas, actividad que se realizaba y descripción del profesional sanitario de la caída. 
En el sistema de información, los profesionales deben de registrar las caídas producidas a partir un cuestionario en el que aparecen los siguientes ítems: Ítems de respuesta abierta. En concreto, estos ítems se asociaron a las variables de actividad realizada, medidas preventivas asumidas y descripción de la caída. Ítems de respuesta cerrada. Se trataba de respuestas asociadas a las variables de número de caídas, causas de las caídas, diagnóstico, tratamiento que se recibía y consecuencias. El cuestionario se acompañaba de un registro observacional que debía ser rellenado por los profesionales sanitarios, y que complementaba la información obtenida.

\section{RESULTADOS}

\section{Caídas, características y factores asociados}

Entre los años 2017 y 2018, se registró un total de 16 caídas, en 8 usuarios del centro residencial. La edad media de las personas que habían padecido la caída era de 87,88 años, correspondiéndose población sobreenvejecida.

Figura 1. Tiempo de permanencia en el centro residencial

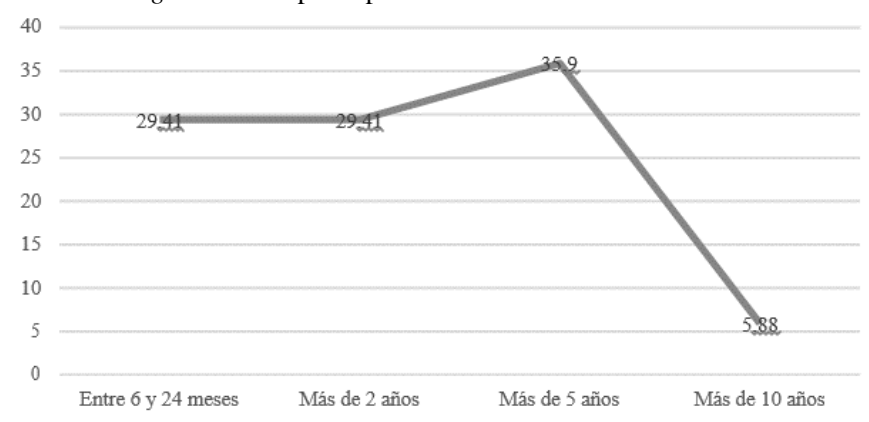

En concreto, el valor mínimo fue de 80 años y el valor máximo fue de 95 años. Las caídas predominaron entre el sexo femenino, representando un 62,5\% de las caídas. Además, los usuarios que habían padecido una caída presentaban diferentes tipologías de enfermedades o patologías; un $12,5 \%$ estaba diagnosticado de enfermedades cardiovasculares, un $12,5 \%$ de enfermedades del sistema nervioso y un $62,5 \%$ sufría deterioro cognitivo. Los tratamientos farmacológicos más frecuentes que tomaba la muestra analizada eran en un 50\% de los casos ansiolíticos y en un 37,5\% hipotensores.

Un aspecto relevante es que, aunque, la retirada de las sujeciones se realizó durante los últimos dos años, muchos usuarios, llevaban más años en el centro residencial. En concreto, en un 35,29\% de las caídas los usuarios llevaban más de 5 años en el centro, un 29,41\% llevaba más de 2 años, un 29,41\% entre 6 meses y 2 años y un 5,88\% más de 10 años (véase figura 1 ). 
Se identifica heterogeneidad entre los factores que predispusieron la caída. Para empezar, asociados a los factores intrínsecos, en un 68,8\% de los casos el motivo principal fue la inestabilidad motora que presentaba la persona mayor. Seguido de este motivo, aparecían estados anímicos nerviosos, agitación o inquietud, siendo el motivo principal en un $18,8 \%$ de las caídas (véase tabla 2). Por otro lado, respecto a los factores extrínsecos, se identificaron que en un $12,5 \%$ fueron debidas a suelo resbaladizo y en un $6,3 \%$ a suelo irregular. No obstante, no se registró ninguna caída provocada por la falta de restricciones físicas o de barreras de sujeción.

Tabla 2. Porcentajes de las caídas según factor que la predispone

\begin{tabular}{lccc}
\hline Factor intrínseco & Porcentaje & Factor extrínseco & Porcentaje \\
\hline Estado de ánimo nervioso o agitación & 18,8 & $\begin{array}{c}\text { Falta de } \\
\text { restricciones }\end{array}$ & 0 \\
\hline Desorientación & 6,3 & Falta de barreras & 0 \\
\hline Problemas de visión & 0 & Calzado & 0 \\
\hline Inestabilidad motora & 68,8 & Suelo resbaladizo & 12,5 \\
\hline $\begin{array}{l}\text { Consecuencias o problemas causados } \\
\text { por patologías }\end{array}$ & 6,3 & Suelo irregular & 6,3 \\
\hline
\end{tabular}

Por otro lado, en el análisis de las caídas, se debe de establecer qué tipología de actividad provocó la caída. En la mayoría de las ocasiones, las caídas se vinculaban a intentos de movilidad. En concreto, los intentos por levantarse o sentarse en la silla promovieron las caídas (en un $25 \%$ de los casos) o de levantarse o sentarse en la cama (en un $25 \%$ de los casos) (véase tabla 3). También se registraron otras actividades previas a las caídas: caminar con ayuda de aparatos $(12,5 \%)$, caminar solo $(6,25 \%)$, caminar con ayuda de otra persona $(6,25 \%)$ o intentar coger un objeto elevado $(6,25 \%)$. Se debe destacar que ninguna de las caídas provocó consecuencias graves. Más concretamente, se observó que un $62,5 \%$ de las caídas no tuvieron consecuencias y un 31,3\% sólo provocó contusiones leves. Si es cierto que, una de las caídas, provocó una herida que si necesitó sutura.

Tabla 3. Porcentajes de las caídas según actividades realizas previas a la caída

\begin{tabular}{lc}
\hline Actividad previa & Porcentaje \\
\hline Intentar levantarse o sentarse en la cama & 25 \\
\hline Intentar levantarse o sentarse en la silla & 25 \\
\hline $\begin{array}{l}\text { Intentar coger un objeto por encima de su } \\
\text { cabeza }\end{array}$ & 6,25 \\
\hline Caminar con ayuda de otra persona & 6,25 \\
\hline Caminar con la ayuda de aparatos & 12,5 \\
\hline Caminar solo & 6,25 \\
\hline No se conoce la actividad que realizaba & 6,25 \\
\hline Otras actividades & 12,5 \\
\hline
\end{tabular}

\section{Medidas alternativas de prevención de caídas}

Como medidas alternativas de prevención de caídas, el centro residencial realizó un plan de intervención individualizado, tal y como se ha mencionado anteriormente. Para cada usuario, se valoró que estrategia era la más adecuada. En 
concreto, para un 31,3\% de las caídas se determinó sólo supervisar y seguir a los usuarios para comprobar y evitar posibles caídas. Los profesionales vigilaban sus actuaciones para identificar posibles situaciones de riesgo. De igual modo, en un 12,5\% de las caídas producidas, se realizó una modificación ambiental, en la que, se substituía el estímulo que había provocado la caída (véase tabla 4).

También, en 4 ocasiones, después de las caídas, se optaba por educar sobre la marcha del usuario, enseñándole otras estrategias para intentar no volver a perder el equilibrio y acabar cayendo. El fortalecimiento de las extremidades inferiores también fue una intervención que se seleccionó tras un $12,5 \%$ de las caídas. Por último, se apostó, tras un $6,3 \%$ de las caídas, por modificar el tratamiento farmacológico de los usuarios.

\begin{tabular}{ll} 
Tabla & \multicolumn{2}{l}{ Porcentajes de las medidas alternativas de prevención de } \\
\cline { 2 - 3 } & \begin{tabular}{ll} 
Medida alternativa de prevención de caídas & Porcentaje \\
\cline { 2 - 2 } Control o supervisión por parte de profesionales & 31,3 \\
\cline { 2 - 2 } Modificación ambiental & 12,5 \\
\hline Reeducación de la marcha (caminar) & 12,5 \\
\hline Fortalecimiento de las extremidades inferiores & 12,5 \\
\hline Múltiples intervenciones & 12,5 \\
\hline Revisión farmacológica & 6,3 \\
\hline Paseos por el centro residencial & 0 \\
\hline Valoración de protocolos de sujeciones & 0 \\
\hline Derivación a especialista & 0 \\
\hline Uso de ayudas dinámicas & $\underline{0}$ \\
\hline
\end{tabular}
\end{tabular}

\section{CONCLUSIONES/DISCUSIÓN}

El temor a las consecuencias vinculadas con las caídas en personas mayores ha provocado que se establezcan las restricciones físicas como medida de actuación. Aún hoy en día, las restricciones son una práctica actual en centros de día y residencias.

No obstante, cada vez son más, los estudios que apuntan a que la eliminación de las sujeciones, siempre y cuando, se substituyan por medidas alternativas, no tienen por qué implicar consecuencias severas (Burgueño y Heras, 2017; De Bellis et al., 2013; Villacieros et al., 2013). De hecho, las restricciones pueden conllevar más repercusiones que las que implicarían las caídas (Hamers y Huizing, 2005; Mion et al., 2011).

Efectivamente, mediante el presente estudio, se verifica que las consecuencias no son graves. A pesar de que se registran 16 caídas (en 8 usuarios), en un $62,5 \%$ no tuvieron consecuencias severas y en un 31,3\% sólo aparecieron algunas contusiones. Además, las caídas se producen en grupos de edad avanzada (media de edad de 87,88 años) y que presentan diagnósticos de enfermedades relevantes. Específicamente, un $62,5 \%$ padecía un deterioro cognitivo y un $25 \%$ alguna enfermedad cardiovascular o del sistema nervioso.

La inestabilidad y los estados nerviosos en la persona mayor eran los motivos más frecuentes de caídas (en un $68,8 \%$ y un $18,8 \%$ de las caídas, respectivamente). Como 
estrategias alternativas, se apostaba, específicamente, por paliar las causas que provocaban las caídas, como se explicaba anteriormente. Asociada a los factores internos, se fomentaba, sobretodo, por el fortalecimiento de las extremidades inferiores y por la educación de la marcha (en un $25 \%$ de los casos). En cambio, como factores externos destacaba el suelo resbaladizo o irregular, provocando que, como medida alternativa, se apostara por la modificación ambiental (12,5\%) o la supervisión por parte de los profesionales $(31,3 \%)$.

Cabe destacar que, además de las medidas anteriormente mencionadas, en el centro residencial gestionado por Intress, también se proporcionaban otras actuaciones menores que también se dirigían a atenuar las consecuencias de las caídas. Asociadas con las deambulaciones, se trabajaba con el fisioterapeuta, reforzando el equilibrio, usando e incorporando diferentes caminadores y fortaleciendo la musculatura también superior. Además, se incorporan mecanismos tecnológicos, como sensores de movimiento, camas articuladas, luces automáticas, etc.

Sin duda, esas medidas deben ser tenidas en cuenta en cualquier programa de retirada de sujeciones físicas. Además, programas eficaces, según señala De Bellis et al. (2013), también deben incorporar los siguientes aspectos: a) uso de protectores de caderas en personas con riesgo a fractura de caderas, b) incremento de dispositivos asistenciales que palien y actúen como refuerzo ante problemas físicos y sensoriales, c) tratamiento de enfermedades o patologías que tienen un mayor riesgo de caídas (déficits visuales, patologías óseas, etc.), d) eliminación de barreras arquitectónicas y e) programas grupales comunitarios que favorezcan la educación y la sensibilización ante posibles prevenciones de caídas, como el taichí. Para conseguir las estrategias o las intervenciones anteriores, se debe apostar por una normativa y legislación que ampare el proceso de adaptación y que mejoren la autonomía del paciente, orientados a que se desarrollen un mayor número de Actividades de la Vida Diaria (AVD) (De Bellis et al., 2013). En definitiva, la eliminación de restricciones físicas se debe establecer como un criterio de valoración vinculado a niveles de calidad de los centros residenciales.

Algunos autores van más allá, señalando que la retirada de sujeciones mejorías en el número de caídas tras la retirada de sujeciones. Sin embargo, en este estudio no se ha podido demostrar por la limitación de la muestra. En cualquier caso, si se debe subrayar que la retirada de sujeciones no empeora el número de caídas producidos. La reducida muestra de usuarios sería una de las principales limitaciones que presenta el estudio. Sin duda, otro aspecto clave en la adecuada eficacia de la retirada de sujeciones físicas es que los formadores posean formación específica. No obstante, esa formación no debe englobar únicamente el proceso de aplicación del protocolo, sino, que, además, debe conllevar otros requisitos como los valores éticos o el bienestar emocional de la persona mayor (Tortosa et al., 2016). 
En futuras líneas de investigaciones, se debería atender, precisamente, a esas dos limitaciones. Por un lado, se debería conseguir una muestra representativa de la población, que permita la generalización de los resultados. Además, se debería apostar por una metodología experimental, compuesta por grupo experimental y control. Esa estrategia permitiría el contraste de hipótesis, verificando o refutando si la retirada de sujeciones incrementa el número de caídas. Este último aspecto será esencial para poder defender la retirada de sujeciones como una actuación óptima y formar parte de un protocolo de buenas prácticas.

\section{REFERENCIAS}

Briones, M.A., y Rodríguez, M.B. (2017). Efectividad de las intervenciones dirigidas a reducir las sujeciones físicas en personas mayores de 65 años institucionalizadas: una revisión sistemática. Revista Española de Geriatría y Gerontología, 52(2), 93-101.

Burgueño, A.A., y Heras, C. (2017). Centros libres de sujeciones físicas: un estándar de oro de calidad. Revista Argentina de Gerontología y Geriatría, 31(3), 77-82.

Carballo, A., Gómez, J., Casado, I., Ordás, B., y Fernández, D. (2018). Estudio de prevalencia y perfil de caídas en ancianos institucionalizados. Gerokomos, 29(3), 110-116.

De Bellis, A., Mosel, K., Curren, D., Prendergast, J., Harrington, A., y Muir, E. (2013). Education on physical restraint reduction in dementia care: a review of the literature. Dementia, 12(1), 93-110.

Hamers, J.P., y Huizing, A.R. (2005). Why do we use physical restraints in the elderly? Zeitschrift für Gerontologie und Geriatrie, 38(1), 19-25.

Mion, L.C., Sandhu, S.K., Khan, R.H., Ludwick, R., Claridge, J.A., Pile, J., y Winchell, J. (2010). Effect of situational and clinical variables on the likelihood of physicians ordering physical restraints. Journal of the American Geriatrics Society, 58(7), 1279-1288.

Sánchez, E.S., Guiteras, P.B., Llanes, J.M., Bustos, A.M., Beneytoa, V.M., y Gaju, R.T. (2004). Las caídas en los mayores de 65 años: conocer para actuar. Atención Primaria, 34(4), 186192.

Santamaría, A.L., Giménez, P.J., Satorra, T.B., Orrio, C.N., y Montoy, M.V. (2015). Prevalencia y factores asociados a caídas en adultos mayores que viven en la comunidad. Atención Primaria, 47(6), 367-375.

Tortosa, M.A., Granell, R., Fuenmayor, A., y Martínez, M. (2016). Efectos de un programa de eliminación de sujeciones físicas sobre personas mayores con demencia en residencias. Revista Española de Geriatría y Gerontología, 51(1), 5-10.

Villacieros, M., Steegman, L., Mejías, M.L., y Bermejo, J.C. (2013). Diseño y validación de una escala de actitudes de los profesionales asistenciales hacia la liberación del uso de sujeciones en personas dependientes. Anales del Sistema Sanitario de Navarra, 36(3), 407-418.

World Health Organization (2008). Who global report on falls prevention in older age. World Health Organization. Ageing, y Life Course Unit.

Recibido: 06 de septiembre de 2019

Recepción Modificaciones: 10 de octubre de 2019

Aceptado: 12 de octubre de 2019 